\title{
COMPUTING ON MASKED DATA TO IMPROVE THE SECURITY OF BIG DATA
}

\author{
Vijay Gadepally, B. Hancock and B. Kaiser, J. Kepner, P. Michaleas, M. Varia, A. Yerukhimovich \\ MIT Lincoln Laboratory, Lexington, MA 02420 \\ \{vijayg, braden.hancock, benjamin.kaiser, kepner, pmichaleas, mayank.varia, arkady\}@ 1l.mit.edu
}

\begin{abstract}
Organizations that make use of large quantities of information require the ability to store and process data from central locations so that the product can be shared or distributed across a heterogeneous group of users. However, recent events underscore the need for improving the security of data stored in such untrusted servers or databases. Advances in cryptographic techniques and database technologies provide the necessary security functionality but rely on a computational model in which the cloud is used solely for storage and retrieval. Much of big data computation and analytics make use of signal processing fundamentals for computation. As the trend of moving data storage and computation to the cloud increases, homeland security missions should understand the impact of security on key signal processing kernels such as correlation or thresholding. In this article, we propose a tool called Computing on Masked Data (CMD), which combines advances in database technologies and cryptographic tools to provide a low overhead mechanism to offload certain mathematical operations securely to the cloud. This article describes the design and development of the CMD tool.
\end{abstract}

Index Terms - Big Data; Accumulo; D4M; Encryption; Information Security

\section{INTRODUCTION}

Recent events, such as [1], highlight the growing need to improve the security of big data stored in the cloud. The traditional challenges associated with big data are often referred to as the 3 Vs of big data: Volume, Velocity and Variety. The sensitivity of data being processed in the cloud is increasing, and it is possible to make use of cryptographic advances to secure processing in the cloud to address a growing 4 th $\mathrm{V}$ of big data - Veracity (the closest word to security that starts with a 'V'). Unfortunately, the volume, velocity and variety properties of big data systems challenge various system components and are often used to justify ignoring this increasingly important factor. In a common computing infrastructure where

This work is sponsored by the Assistant Secretary of Defense for Research and Engineering under Air Force Contract \#FA8721-05-C-0002. Opinions, interpretations, recommendations and conclusions are those of the authors and are not necessarily endorsed by the United States Government sensor data is fed into a shared computing cloud to be used by authorized users, the goal is to determine techniques that allow the potentially untrusted computing cloud to store and perform computation directly on encrypted or masked data.

Large heterogenous organizations, such as the Department of Homeland Security, have a particular need for making use of the cloud. Key features such as resource pooling, rapid elasticity and on-demand self-service [2] provide the ability for diverse organizations to share information efficiently and easily. However, these advantages also create a single point where adversaries may try to attack the confidentiality, integrity and/or availability of sensitive information stored or being processed. As a specific example, a recent report by the Department of Homeland Security (DHS) looked at the viability of making use of cybersecurity insurance (http://www.dhs.gov/publication/cybersecurity-insurance).

One of the requirements for evaluating the applicability of such a system will be in the ability to track cyber incident reports in a central location such as a commercial cloud offering. This data stored in the repository may provide adversaries with information that could be used to reverse engineer the details of a cyber security breach [3]. In this example and many more, cryptographic protections can be used to mitigate the risks of data being stored and processed in the cloud.

Prior work has looked at various techniques to improve the confidentiality, integrity and availability of big data systems and an overview is provided in [4]. There are many existing cryptographic tools capable of performing some level of computation on encrypted data. One of these is Fully Homomorphic Encryption (FHE), first introduced in 2009 [5]. This method allows arbitrary analytic computations to be performed on encrypted data while maintaining a strong security guarantee called "semantic security" (also referred to as randomized encryption and is denoted as RND). The current state of the art implementations of this scheme [6, 7] have a minimum computational overhead of $10^{5}$, which removes them from consideration for any practical big data system. Secure Multi-Party Computation (MPC) techniques [8, 9] are another possibility with semantic security guarantees, but these techniques also currently experience excessive overhead and require large amounts of customization for a given application and threat model. 
An alternative solution is to make use of more relaxed forms of encryption that reveal small amounts of information, as first described for the database setting in [10]. In such a system, one may make use of the deterministic encryption method (DET), which encrypts any plaintext and key to a single ciphertext thereby allowing for fast equality calculations to be performed on encrypted data (a property very desirable for database applications). Other relaxed forms of encryption, such as Order Preserving Encryption (OPE) which preserves the relative order of all inputs, provide high functionality in return for relaxed security guarantees. In this article we propose the Computing on Masked Data (CMD) [11] system that uses a combination of RND, DET, and OPE modes to mask sensitive data, insert into a NoSQL database and perform simple computation on the masked data. Further, for many situations, data integrity (not necessarily confidentiality) is a large concern and we provide a 4th masking level - Authentication (AUT). The article is structured as follows. Section 2 provides an overview of the proposed tool. Section 3 describes the implementation of the CMD tool. Section 4 describes how CMD can be applied to simple signal processing kernels which are important to analytic developers. Section 5 discusses the performance of CMD on these signal processing kernels. Finally, in Section 6 we discuss potential CMD applications and conclude.

\section{COMPUTING ON MASKED DATA}

Associative Arrays, the data type provided by the Dynamic Distributed Dimensional Data Model (D4M) [12], are used as the base schema to represent unstructured large data sets. The D4M schema encapsulates all semantic information of a dataset into the rows and columns of a sparse associative array. We make use of this property to use various different encryption techniques to mask the rows and columns depending on required functionality. An overview of the schema is described in Figure 1 .

Associative array rows, columns and values are masked through the CMD mask function, which encrypts entities of the sparse associative array. For example, if one intends to perform range operations on columns, columns may be masked using OPE. Associative arrays support the full range of linear algebra operations such as addition, multiplication, etc. A more thorough description of the CMD system is provided in [11].

\section{CMD IMPLEMENTATION}

A prototype of CMD has been developed as a MATLAB/GNU Octave toolbox. The current version of CMD allows a user to select among four masking levels: RND, DET, OPE and AUT. A combination of masking levels can be used across the rows, columns and values of the associative array representation. Selecting a masking level depends upon the desired

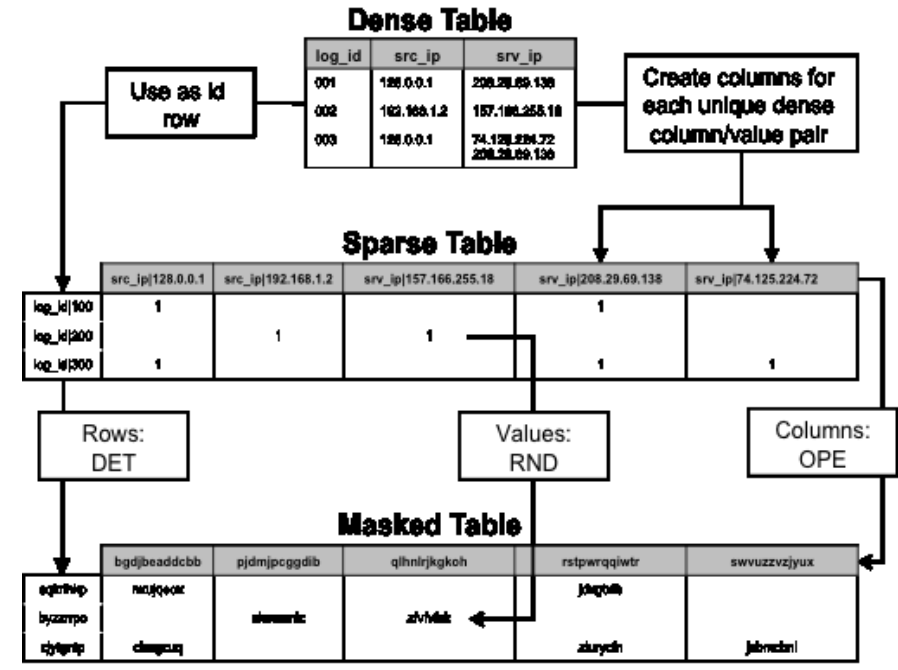

Fig. 1: Masking illustrative network data records. Dense data is made sparse using the D4M schema and moves semantic information to the rows and columns of sparse assosciative arrays. The sparse table is then masked using a variety of encryption schemes depending on the desired functionality. The terms DET, OPE and RND are described in Section 3 .

\begin{tabular}{|l|l|l|}
\hline $\begin{array}{l}\text { Masking } \\
\text { Level }\end{array}$ & Information Leaked & $\begin{array}{l}\text { Database Func- } \\
\text { tionality }\end{array}$ \\
\hline RND & $\begin{array}{l}\text { Hides all information } \\
\text { about data }\end{array}$ & Decrypt only \\
\hline DET & Reveals equality patterns & Query plaintext \\
\hline OPE & $\begin{array}{l}\text { Reveals equality patterns } \\
\text { and order }\end{array}$ & $\begin{array}{l}\text { Plaintext range } \\
\text { query }\end{array}$ \\
\hline AUT & $\begin{array}{l}\text { Reveals everything but } \\
\text { protects data integrity }\end{array}$ & All queries \\
\hline
\end{tabular}

Table 1: Summary of security provided and database functionality for CMD masking levels

functionality. A summary of database (DB) functionality and security afforded for different masking levels is provided in Table 1. The implementation of each of the encryption techniques is described below.

\subsection{Semantically Secure Encryption - RND}

The CMD implementation of RND uses the AES-256 block cipher found in OpenSSL (www.openssl.org) in the Cipher Block Chaining or Galois Counter Mode. The cryptographic key is derived from a user-provided password and an 8-byte salt using 1000 rounds of the derived key generation loop. Each ciphertext has a minimum length of one AES block and is derived from both the cryptographic key and an Initialization Vector (IV) of 16 bytes. The IV is generated using the OpenSSL command RAND_bytes, which generates an arbi- 


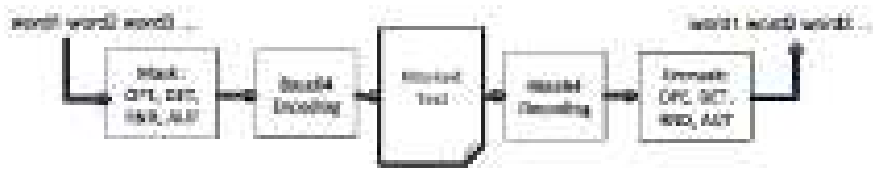

Fig. 2: Plaintext data is masked using a combination of possible modes. Masked data is then encoded for storage purposes. Accessing the data involves first decoding the stored masktext and unmasking.

trary length string of cryptographically strong pseudo-random bytes. This step ensures that each masking of the same plaintext will yield a different ciphertext, thereby protecting equality information. To simplify string handling, ciphertexts are converted into printable characters using Base64 encoding. An overview of the process is described in Figure 2

\subsection{Deterministic Encryption - DET}

The implementation of DET is identical to that of RND except for the way that the IV is generated for each message. To leak only equality information and nothing more, DET masking requires that each message uses an IV that is unique to that message and deterministically obtained. This is achieved in CMD by using the SHA- 1 hash of each message as its own IV, truncated to 16 bytes. The OpenSSL SHA-256 implementation may be substituted for SHA-1 if more security is desired, but this comes with an approximate $40 \%$ increase in computation time compared to using the SHA-1 hash (which can perform approximately $1.7 \times 10^{6}$ hashes/second).

\subsection{Order Preserving Encryption - OPE}

For the OPE masking level, a mutable scheme called mOPE, initially proposed in [13], was adopted. The mOPE model consists of a trusted client and an untrusted server that interact with each other. The untrusted server is never given access to any plaintext values or the user password, and the trusted client is never required to store or analyze the entire data set at once. The data are stored on the server in a binary search tree as ciphertexts (obtained through DET encryption operations on the client). Because ciphertexts do not leak order information, the server must communicate with the client through an interactive session to determine the correct location in the binary search tree for each ciphertext. Starting at the root of the tree, the server sends the client the ciphertext of the current node. The client decrypts the ciphertext at that node, compares it to the plaintext being inserted, and returns a 0 (left) if the plaintext is less than the value at that node or a 1 (right) if the plaintext is greater. The new current node is then returned, and the process repeats. When the exact location of the plaintext to be inserted has been found, the client sends the server the ciphertext of that value along with a command to insert it there in the tree. The OPE ordertext representa- tion of a given plaintext is simply the path to its ciphertext in the binary search tree, concatenated with padding of a 1 and a sufficient number of 0 s to make all ordertexts the same size. We once again chose to use a default size of 16 bytes (thereby allowing for 216 entries if $0 \mathrm{~s}$ and $1 \mathrm{~s}$ are stored as strings or 2128 entries if the path is stored as bits). Figure 3 describes a sample OPE Tree and corresponding OPE Table, which indicates the ordertext value for each ciphertext in the tree. Querying for data (or a range of data) occurs by determining the position of the masked data.

\subsection{Authentication mode - AUT}

The fourth supported operation available in CMD is AUT which stands for Authentication. This operation does not actually mask the data at all and plaintext is left as plaintext. Prepended to the plaintext, however, is a hash-based message authentication code (HMAC). Here too, we prefer to use SHA-1 for performance reasons. When a given message is unmasked with AUT, the HMAC that is stored with the plaintext can be extracted and compared against a new HMAC of the plaintext message and key. If the two are equal, the user can have confidence that the original message has not been modified since it was first stored as generating the same HMAC requires knowledge of the key. While the AUT mode does not ensure data confidentiality, it does ensure data integrity.

\section{SIGNAL PROCESSING WITH CMD}

In this section, we demonstrate the applicability of CMD on some basic signal processing kernels. Given the relationship between the associative array representation and graphs, we pay particular attention to correlation and thresholding, which can be used as building blocks to construct a variety of linear algebra and signal processing algorithms.

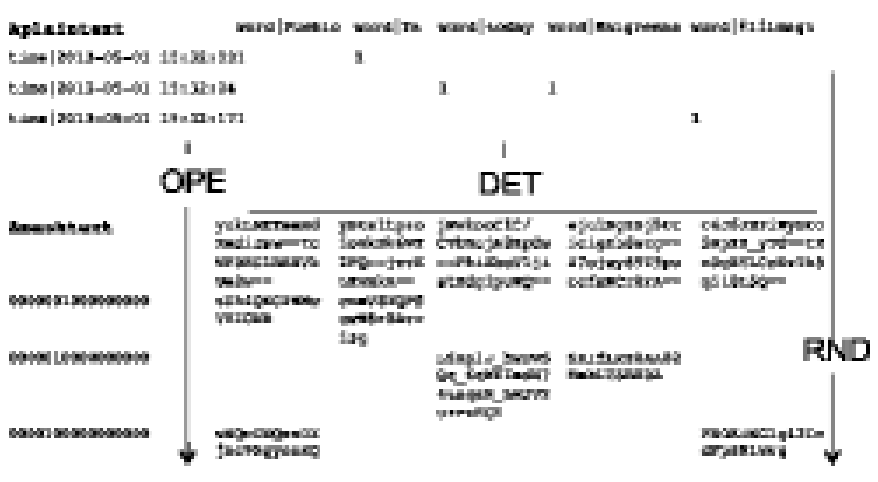

Correlation provides a measure of the statistical relationship between two entities. Calculating the correlation of two associative arrays involves transposing and multiplying. Since CMD preserves the associative array structure of big data, we can perform masked correlation by multiplying two 


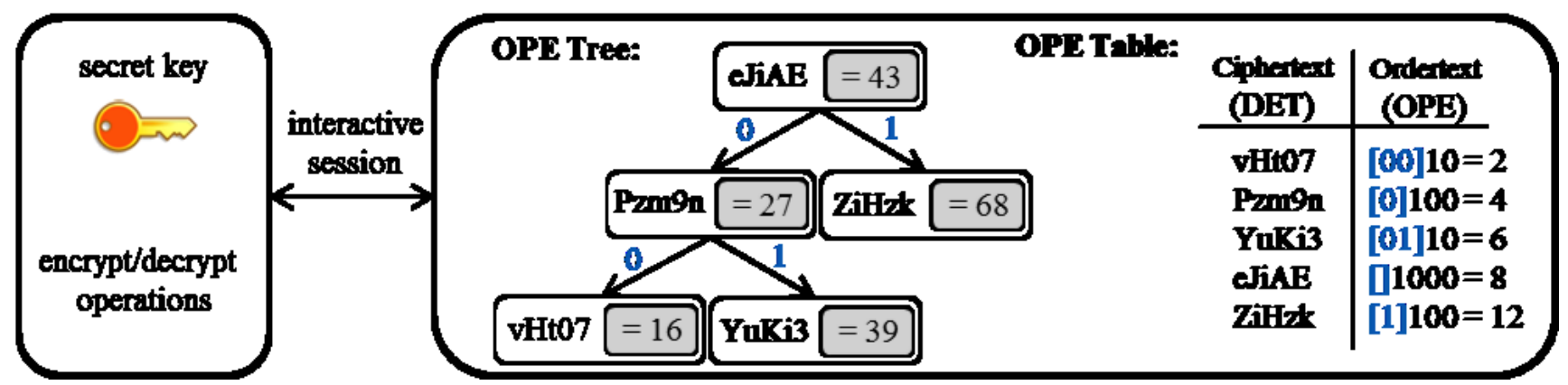

Fig. 3: Overview of the data structures in the mOPE scheme. Each node in the OPE tree contains a ciphertext. The plaintext value of each ciphertext is shown here in gray blocks for illustration purposes and are not stored in the tree. The ordertext value corresponding to each ciphertext comes from following the path to each ciphertext from the root of the tree. Ciphertext-ordertext pairs are stored in a database to enable fast lookup. Figure concept from [13].

masked arrays which are masked using any of the supported masking modes. This task can be done on a potentially untrusted server. Consider a sample dataset which contains data collected from the social media website Twitter. The data $i$ stored as a tuple that contains a timestamp, unique Twitte identification number (TweetID), and value. A common tas is to find the most common words that exist within the sam tweet as a term of interest, say $t 1$.

CMD can determine the unique TweetIDs by multiplyin the masked version of the dataset Amasktext (abbreviate as Amt) to yield an output associative array Cmasktext (at breviated as $\mathrm{Cmt}$ ):

$\mathrm{Cmt}=\operatorname{Amt}(:, \operatorname{Mask}(t 1))^{\prime} \star \operatorname{Amt}$

Suppose that the term of interest is the word happy which is stored using the D4M schema as word|happy. The above product on the Amt yields the following result:



Unmasking Cmasktext reveals words correlated with word | happy:

Cpt =Unmask (Cmt, ' $\left.\mathrm{DET} \mathrm{T}^{\prime}, \mathrm{DET}^{\prime}, \mathrm{DNND}^{\prime}\right)$

$\begin{array}{llllll}\text { Cplaintext } & \text { word|birthday } & \text { word|others } & \text { word|the } & \text { word|zitaoooo! } & \text { word|zpna } \\ \text { word|happy } & 28 & 46 & 190 & 2 & 1\end{array}$

The second signal processing kernel currently supported is thresholding. Thresholding keeps all values that satisfy a certain criteria and discards the rest. In the example above, we may perform a thresholding to find only common values. For example:

Cpt $=$ Unmask $\left(\mathrm{Cmt}>20,{ }^{\prime} \mathrm{DET} \mathrm{T}^{\prime}, \mathrm{DET}^{\prime}, \mathrm{DNND}^{\prime}\right)$
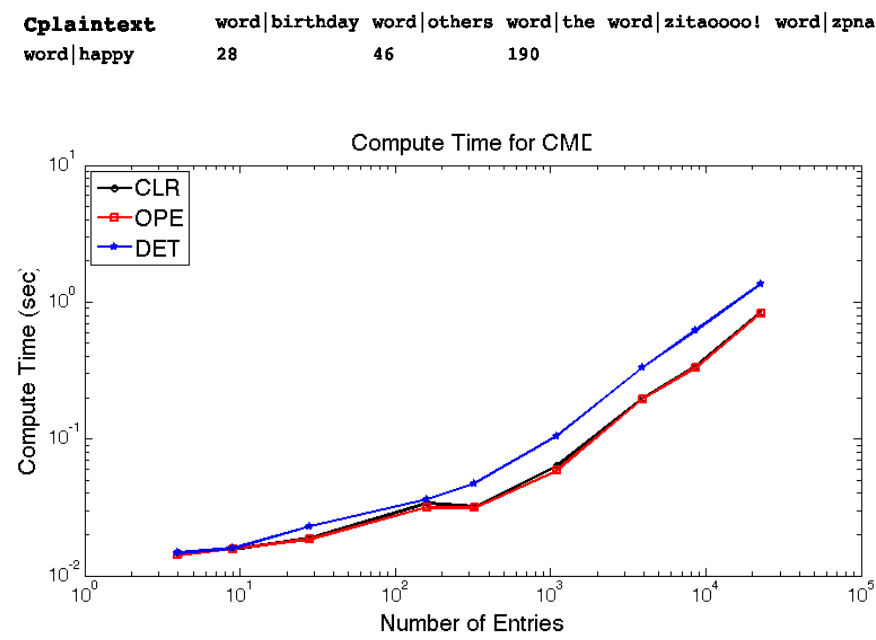

Fig. 4: Performance of different masking levels in performing a correlation of two arrays with varying number of elements

\section{PERFORMANCE RESULTS}

This section describes the performance of the CMD system on varying sizes of input data to quantify the relative performance overhead incurred by performing computation on masked data. For these examples, data is stored in an Apache Accumulo NoSQL database and computation and data retrieval times are presented. One key metric for CMD is the performance overhead incurred with computation on masked data. Figure 4 describes the time taken to compute a correlation of two associative arrays with varying number of entities. As the performance chart shows, the computational overhead involved with using either DET or OPE modes of encryption is minimal (within $2 \mathrm{x}$ ) when compared to performing computation on the plaintext data (denoted by CLR). Performing thresholding on masked data incurs almost no overhead. 
Figure 5 describes the performance overhead incurred in querying and unmasking data masked using different levels of encryption. From this figure, it is clear that the overhead incurred by using different modes of encryption is similar to that of correlation (within $2 \mathrm{x}$ ) when compared to simply retrieving plaintext data (denoted by CLR).

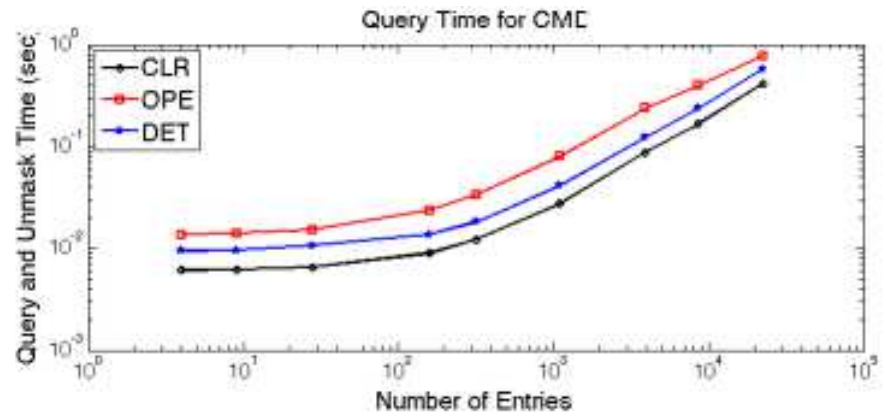

Fig. 5: Query and unmask performance time with varying number of entries

While it is difficult to quantify the reduction in information leakage when compared to plaintext data, the description of leakage in Table 1 shows that the CMD system greatly reduces leakage for applications in which leakage of equality patterns or order are acceptable.

\section{DISCUSSION, CONCLUSIONS AND FUTURE WORK}

Data veracity is a growing concern amongst the big data community. The volume, velocity, and variety, however, pose large challenges to the development of systems capable of protecting the privacy of big data. These challenges are especially evident in organizations that operate large computing clouds to be shared by heterogeneous organizations often with varying data policies. The Computing on Masked Data tool presented in this article can be used in such environments. One organization that exemplifies such an environment is the Department of Homeland Security which is especially prone to such challenges due to their distributed, multi-agency, and time sensitive nature.

Homeland security agencies are increasingly looking to cloud solutions in order to make use of key characteristics such as on-demand self-service, broad-network access, resource pooling, rapid elasticity, and measured service [2]. Commercial entitites such as Amazon have responded to this need by providing US based solutions such as the Amazon GovCloud [14].

The Computing on Masked Data tool described in this article can provide an extra layer of security with minimal impact to performance to individuals or organizations who wish to make use of cloud computing offerings without completely trusting the provider. Consider an example in which different agencies of the DHS wish to maintain a database of security related data such as agent field reports. In order to ensure that this data can be accessed by different agencies, perhaps geographically distributed across the United States, the DHS may wish to make use of a commercial cloud provider such as Amazon Web Service (AWS). While offerings such as GovCloud provide a baseline of security, one may not want to offload data confidentiality protection to a third party via service level agreements and instead make use of cryptographic protections on the data (thus protecting it from unauthorized users from the untrusted cloud provider or other cloud tenants). Using CMD, generated field reports can be encrypted at individual clients and uploaded to the commercial cloud in encrypted form using the Mask command. Simple computation such as correlation or thresholding (suppose to correlate a name across reports from different agencies) can be done directly in the cloud without compromising the confidentiality of requested data. Finally, correlated data can be queried and returned to an authorized end user who can use the Unmask command on the data using a valid key to see the end result. Of course, there are still challenges associated with such tools. For example, organizations may need to agree upon the desired functionality in order to use the same choice of masking levels. Such a challenge may be overcome using a layered encryption scheme as described in [10]. Another challenge is with the efficient management of keys and requires a solution as proposed in [15].

Homeland security researchers and scientists should be aware of the increasing value of data in the cloud and determine methods to mitigate the risk associated with unauthorized disclosure. In this article, we propose a tool - Computing on Masked Data - that is a high performance NoSQL database interface that stores data masked by a choice of techniques. The CMD system supports a wide variety of masking modes and exhibits high performance for common signal processing kernels such as correlation and thresholding. The performance and simplicity of the CMD tool make it an ideal candidate for many applications.

\section{ACKNOWLEDGEMENTS}

The authors would like to acknowledge the anonymous reviewers, Nabil Schear, Rob Cunningham, and the LLGrid operations team at MIT Lincoln Laboratory for their support in developing and testing the CMD prototype. 


\section{REFERENCES}

[1] "What Jennifer Lawrence can teach you about cloud security," ArsTechnica: http://arstechnica.com/security/2014/09/what-jenniferlawrence-can-teach-you-about-cloud-security/.

[2] Timothy Mell, Peter; Grance, "The NIST definition of cloud computing," Special Publication 800-145.

[3] National Protection and Programs Directorate, Department of Homeland Security, "Insurance industry working session readout report," Insurance for CyberRelated Critical Infrastructure Loss: Key Issues.

[4] Sophia Yakoubov, Vijay Gadepally, Nabil Schear, Emily Shen, and Arkady Yerukhimovich, "A survey of cryptographic approaches to securing big-data analytics in the cloud," in the Proceedings of High Performance Extreme Computing Conference (HPEC), 2014.

[5] Craig Gentry, "Fully homomorphic encryption using ideal lattices.," in Symposium on Theory of Computing (STOC), 2009, vol. 9, pp. 169-178.

[6] Shai Halevi and Victor Shoup, "Algorithms in HElib," IACR Cryptology ePrint Archive, vol. 2014, pp. 106, 2014.

[7] Henning Perl, Michael Brenner, and Matthew Smith, "Poster: an implementation of the fully homomorphic smart-vercauteren crypto-system," in Proceedings of the 18th ACM conference on Computer and communications security. ACM, 2011, pp. 837-840.

[8] Michael Ben-Or, Shafi Goldwasser, and Avi Wigderson, "Completeness theorems for non-cryptographic fault-tolerant distributed computation," in Proceedings of the twentieth annual ACM symposium on Theory of computing. ACM, 1988, pp. 1-10.

[9] Andrew C Yao, "Protocols for secure computations," in 54th IEEE Annual Symposium on Foundations of Computer Science. IEEE, 1982, pp. 160-164.

[10] Raluca Ada Popa, Catherine Redfield, Nickolai Zeldovich, and Hari Balakrishnan, "Cryptdb: protecting confidentiality with encrypted query processing," in Proceedings of the Twenty-Third ACM Symposium on Operating Systems Principles. ACM, 2011, pp. 85-100.

[11] Jeremy Kepner, Vijay Gadepally, Pete Michaleas, Nabil Schear, Mayank Varia, Arkady Yerukhimovich, and Robert K Cunningham, "Computing on masked data: a high performance method for improving big data veracity," in the Proceedings of High Performance Extreme Computing Conference (HPEC), 2014.
[12] Jeremy Kepner, Christian Anderson, William Arcand, David Bestor, Bill Bergeron, Chansup Byun, Matthew Hubbell, Peter Michaleas, Julie Mullen, David O'Gwynn, et al., "D4M 2.0 schema: A general purpose high performance schema for the accumulo database," in the Proceedings of High Performance Extreme Computing Conference (HPEC). IEEE, 2013, pp. 1-6.

[13] Raluca A Popa, Frank H Li, and Nickolai Zeldovich, "An ideal-security protocol for order-preserving encoding," in IEEE Symposium on Security and Privacy (SP). IEEE, 2013, pp. 463-477.

[14] "Amazon

GovCloud," http://aws.amazon.com/govcloud-us/.

[15] Roger Khazan and Dan Utin, "Lincoln open cryptographic key management architecture," Tech. Rep., DTIC Document, 2012. 